\title{
LES ENFANTS DE 4 À 6 ANS ET LES INSTITUTIONS CULTURELLES
}

\author{
Sylvie Romano ${ }^{1}$
}

\section{Introduction}

A partir des années 70, l'offre éducative des musées a semblé $s^{\prime}$ intensifier ${ }^{2}$. Les enfants paraissent acquérir peu à peu un véritable statut de visiteur. Aussi, pour répondre à cette demande, de nombreuses institutions culturelles se sont dotées de services éducatifs ou pédagogiques. Ces services ont construit et développé des activités pour les enfants, autour de savoirs parfois scolaires, parfois non formels. C'est aussi dans cette période qu'une sorte de partenariat école/musée s'est développé, en tenant compte à la fois des objectifs et des aspirations de chacun ${ }^{3}$. Ainsi, la visite du musée, illustration d'un cours, a laissé la place à d'autres activités comme celles susceptibles d'apprendre aux enfants à visiter ou à utiliser un musée, à accroître leur sens de l'observation, leur esprit critique...

1 Université d'Avignon. Laboratoire culture \& communication.

2 E. CAIllet, A l'approche du musée, la médiation culturelle, Lyon, Presses universitaires de Lyon, 1995, pp. 201 à 211.

3 F. Buffet (sous la dir. de), Entre école et musée le partenariat culturel d'éducation, Lyon, Presses universitaires de Lyon, coll. "Travaux et Documents", 1998. 
En dehors des services éducatifs, certaines institutions ont pris le parti de se spécialiser dans l'accueil des enfants : le musée en Herbe (à Paris), le Préau des Accoules (à Marseille), le Hellenic Children's Museum (à Athènes) ou encore le Musée des enfants (à Bruxelles)... Parmi ces institutions, deux ont fait l'objet d'enquêtes dont les résultats nous ont permis de recueillir des éléments en matière de pratique d'éducation non formelle et de savoirs informels : la Cité des enfants (espace 3-5 ans) de la Villette et le Préau des Accoules.

Ces deux recherches ont porté exclusivement sur les enfants âgés de 3 à 6 ans. Ce jeune public, en début de scolarisation, ne sait ni lire, ni écrire, ni compter et ne peut donc pas se servir des apprentissages scolaires propres aux savoirs formels ${ }^{1}$. Par contre, ces jeunes enfants sont peut-être réceptifs vis-à-vis d'autres formes de savoirs puisqu'on sait qu'à cette période, ils apprennent vite et beaucoup. En effet, ces tout-petits n'ont pas d'autre choix pour apprendre que d'utiliser les savoirs informels acquis à travers leur vécu (les savoirs formels étant ceux qu'on peut acquérir par l'intermédiaire de l'école). De ce fait, ces très jeunes visiteurs paraissent être un public particulièrement intéressant pour les musées et surtout pour cette recherche.

Ces deux études permettent de faire le point sur la perception réelle qu'ont les enfants de ces deux institutions qui fondent leur fonction éducative sur des démarches différentes de celle de l'école, les savoirs informels, pour atteindre des objectifs similaires et ce, en se basant sur différentes formes de médiations.

La première recherche concerne la Cité des enfants (La Villette, Paris). Elle a été réalisée à la demande de la Cité des sciences et de l'industrie en vue du renouvellement muséographique de la cité des enfants. En effet, depuis leur création, les espaces mis en place n'ont encore jamais été transformés. Dans ce cadre, nous avons réalisé une série d'observations, qui nous a permis d'entrevoir comment les enfants utilisent cet espace basé sur une médiation pro-active, inscrite dans la conception même du parcours, dans celle des écrits d'exposition et dans l'organisation des visites qui implique une certaine autoéducation. Les tout-petits sont obligatoirement accompagnés par un adulte (coéducation parents/enfants) et ne sont pas séparés des autres jeunes visiteurs (coéducation enfant/enfant).

1 L. Filiatrault, Les effets de deux approches éducatives sur l'apprentissage de concepts et la manifestation de comportements illustrant des habiletés intellectuelles chez des enfants de la maternelle visitant un musée, Montréal, Thèse universitaire : Éducation, dirigée par Michel ALLARD, [S.I.] : [S.N.], 1995. 
La seconde recherche, menée au Préau des Accoules (à Marseille), tente d'évaluer, à travers une démarche plus élaborée, l'impact sur le jeune public d'une exposition de peinture. Cette analyse de l'exposition "Camoin au Balcon : un peintre et son monde" et du parcours proposé aux enfants, fournit des éléments originaux sur la façon dont les enfants s'approprient les connaissances qui leur sont transmises. Au Préau, la recherche a porté sur une médiation active qui prend appui sur des activités proposées aux enfants à partir de tableaux authentiques et pas du tout sur des capacités intellectuelles scolaires comme lire, écrire ou compter.

\section{Espace 3-5 ans de la Cité des enfants (La Villette) à Paris}

\subsection{Historique et fonctionmement}

La Cité des enfants est composée de deux espaces, le premier est consacré aux 3-5 ans (il est l'objet de la recherche); le second est réservé aux 6-12 ans. Ces deux lieux correspondent à un découpage par âge et fonctionnent sur le même principe. L'espace 3-5 ans, premières découvertes, de la Cité des enfants a ouvert ses portes en 1992. Situé dans le hall principal de la Cité des sciences, il est à la fois, ouvert sur le monde extérieur puisqu'il est entièrement vitré, et clos pour protéger la tranquillité des enfants lors de leur découverte.

La Cité des sciences et de l'industrie a voulu en faire un lieu d'éveil et de sensibilisation à la science pour les tout-petits. Pour cela, les concepteurs ont aménagé à demeure, sur une surface d'environ $300 \mathrm{~m}^{2}$, différentes installations qui veulent, par l'intermédiaire d'apprentissages non formels, éveiller l'enfant à la connaissance de soi et de son environnement. Cet espace est structuré en 8 sous-parties dans lesquelles il y a plusieurs installations qui offrent des activités aux enfants. Ainsi, au cours de leur visite, les enfants découvrent :

- l'espace percevoir et agir,

- l'espace découvrir son image,

- l'espace règles du jeu,

- l'espace histoire d'un grain de blé,

- l'espace les mains dans l'eau,

- l'espace si j'étais un animal,

- l'espace des petits mécaniciens,

- l'espace du chantier. 
Ces espaces fonctionnent tous de manière autonome. Le sens de la visite est suggéré à travers un parcours libre (sans guide). En son sein, seul le personnel de surveillance et de sécurité est admis, mais aussi les parents et les accompagnateurs dont la présence est obligatoire. Il y a autant d'enfants en compagnie de leurs parents que de groupes scolaires. Et, c'est la seule curiosité des enfants qui doit les guider dans leur découverte. Pendant une heure trente, chaque enfant visite à son rythme ${ }^{1}$ et ensuite il laisse sa place à un autre.

Toutes les activités proposées à l'enfant sont construites à partir de sujets familiers. Elles prennent appui sur le quotidien, comme la voiture ou la météo ; ce qui peut les attirer comme les animaux, un chantier ; ou encore, ce qui est susceptible de les intéresser, comme l'eau. Les jeux, les expériences... sont entièrement pensés pour les enfants appartenant à la tranche d'âge 3-5 ans. Ainsi, chaque espace est conçu à leur échelle (taille, force,...) avec très peu d'écrits. Et surtout, tout est fait pour que chaque enfant, au cours de son parcours, découvre en s'amusant et en expérimentant, de sorte qu'apprendre devienne, en ce lieu, un véritable plaisir...

\subsection{Objectifs de l'institution}

Lors de la conception de cet équipement, une équipe de spécialistes de la petite enfance a été consultée. Elle était composée de personnes d'horizons différents : comités de quartier, personnel de crèches, de haltes garderies, assistantes maternelles, écoles nouvelles...

Les objectifs de ces spécialistes de la petite enfance et de la Cité des sciences et de l'industrie étaient multiples :

- mettre à la disposition des enfants un espace d'éveil et de sensibilité à la science en leur donnant un lieu où ils pouvaient expérimenter les choses ;

- offrir aux enfants un espace qui puisse les rendre libres et autonomes, un espace où chacun peut découvrir à son rythme ${ }^{2}$; - créer un espace de socialisation et d'échange entre les enfants ;

1 B. Poucet, M. Van Praet, "Les musées, lieux de contre-éducation et de partenariat avec l'école", Éducation et Pédagogie, n 16, Paris, 1992, pp. 21-29. M. AllARD, "Le musée comme lieu d'apprentissage", Vie pédagogique, n 84 ,

2 Québec, mai-juin 1993, pp. 41-43.

Ibid. 
- enfin concevoir un espace basé sur la coéducation, un espace où on découvre ensemble, au cours d'un moment privilégié, entre parents et enfants mais aussi un espace où on s'éduque entre pairs, d'enfant à enfant, un espace où des liens particuliers peuvent se tisser.

\subsection{Enquête et résultats}

Cette étude a permis d'explorer deux axes différents : - d'une part, la manière dont les enfants ressentent le site par rapport aux objectifs d'apprentissages non formels (de socialisation, d'autonomisation, en prenant appui sur la coéducation) que s'étaient fixés les concepteurs ;

- d'autre part, les relations que peuvent développer les visiteurs adultes et enfants à travers ce type de dispositif.

Pour ce faire, différentes méthodes ont été utilisées : suivis de parcours à distance afin de savoir si certaines portions du parcours étaient évitées ou, au contraire, "sur-utilisées"; entretiens semidirigés tant avec les enfants, qu'avec les adultes pour connaître les préférences des enfants et des parents pour les différents espaces; et parcours accompagnés pour mieux cerner la façon dont les visiteurs, enfants et adultes, utilisaient cet espace (lorsque les parents ont accepté de partager leur visite avec une personne étrangère à la cellule familiale)...

Certaines remarques et réflexions de parents et d'enfants semblent aller dans le sens des objectifs visés par les concepteurs de la Cité des enfants. Ainsi, dès leur entrée dans chaque espace, nombre d'enfants semblent attirés par ce lieu. Pour s'en convaincre, il suffit d'observer les espaces tels que percevoir et agir ou encore les mains dans l'eau. Les enfants agissent instinctivement, et ce lieu les attire car il les sollicite sans cesse ${ }^{1}$. Les savoirs n'y sont pas figés; ils s'acquièrent en faisant appel à leurs sens, en bougeant, en écoutant, en ressentant les choses. Tout ceci tend à montrer un certain engouement, sinon pour les sciences, en tout cas pour la manipulation associée.

Les jeunes enfants poussent parfois même cet exercice plus loin que ce que les concepteurs ne l'avaient prévu. Ils ont souvent une

1 E. CAILlet, O. CoPPEY, "Le faire savoir ou la délectation au musée", in E. FAUBLEE, En sortant de l'école... musées et patrimoine, Paris, Éd. Hachette Éducation, Centre de Documentation Pédagogique, coll. "Ressources Formation", 1992, pp. 103 à 110 . 
vision divergente de celle des créateurs de l'espace. Dans le début de la visite, un cours d'eau a été installé afin de matérialiser le parcours que les enfants doivent suivre. Sur ce cours d'eau, flottent de petits bout de mousse de formes et de couleurs différentes afin d'indiquer le sens de la visite.

Or, en fait de signalisation, ce mécanisme a été complètement détourné par les enfants qui s'amusent à repêcher ces petits bouts de mousse, à les classer... ; ce cours est devenu à lui seul une manipulation. Une mère témoigne : "ma fille (...) a trouvé des choses à faire avec ce ruisseau (...) ; au début c'était ça, prendre un petit jouet là, (...) le remettre plus bas dans l'eau, et après elle les a tous sortis de l'eau et les a mis sur les petits ponts, là elle les assemblait par formes et par couleurs". Cette enfant n'est pas isolée ; ce cours d'eau est devenu une manipulation grâce à laquelle on s'initie aux formes et aux couleurs d'une manière distrayante.

De même, il semble possible de penser que cet espace offre aux enfants la possibilité d'être libres et autonomes. Ainsi, dans l'espace grain de blé, un père qui veut aider son enfant à tourner les manivelles, s'est vu mis à l'écart par l'enfant irrité par cette intrusion dans son jeu : "C'est moi qui le fais !"; ou encore, cet enfant qui explique ce qu'il a fait : "j'ai fait des travaux, j'ai fait la voiture..."; et cet autre de dire très sérieusement en montrant le chantier (installation où les adultes sont totalement interdits) : “j' ai été au boulot".

Dans le cadre d'un espace de socialisation', la réflexion d'un enfant faite à ses camarades de classe et à d'autres enfants qui se trouvent dans l'espace grain de blé semble aussi démontrer que des interactions se concrétisent. Moitié tyrannique, moitié encourageant. Il crie : "eh, vous pouvez tourner, j' ai rien là... Là très bien Brian c'est super, merci, vas-y encore... S'il vous plaît du blé, encore du blé, alors Brian !!". Il est clair que cet espace, conçu pour privilégier le travail de groupe, semble jouer ici son rôle. En allant plus loin, on peut penser qu'il révèle jusqu'aux rapports de force que les enfants peuvent établir au sein d'un groupe. Cet enfant est en quelque sorte un "leader" : organisant le travail et obligeant tous les enfants à participer. Ce cas de figure se retrouve aussi dans un espace comme le chantier.

1 P. Champy, C. Eteve, Dictionnaire encyclopédique de l'éducation et de la formation, Paris, Nathan Université, coll. "REF", 1994. 
Enfin, pour ce qui est de la coéducation, la plupart des enfants lorsqu'ils sont primo-visiteurs, ont besoin de s'appuyer de temps en temps sur un adulte afin de mieux comprendre ce qu'ils doivent faire. C'est le cas par exemple, dans l'espace règles du jeu occupé par des ordinateurs. La coéducation parent/enfant fonctionne ici de façon plus évidente. Elle crée un moment privilégié. Cette coéducation fonctionne aussi parfois entre pairs. Ainsi, dans l'espace percevoir et agir, l'installation du trajet surprise permet de tels moments. Une petite fille de 2 ans et demi a peur de passer un barrage formé par 2 gros rouleaux ; un petit garçon de 6 ans qui joue dans le trajet depuis un moment a fini par la prendre par la main pour passer avec elle. Aucun mot n'a été échangé ; les enfants ne se connaissaient même pas et se sont séparés tout de suite après, mais la petite fille est retournée immédiatement dans le trajet pour réessayer toute seule. De même, on peut citer sur l'espace si j'étais un animal, la réaction d'un petit garçon ravi qui appelle les autres enfants : "Venez voir, venez voir ! Venez voir les trucs là avec un petit labyrinthe !... Venez voir la petite souris"... les autres enfants arrivent.

Tous ces exemples évoquent des apprentissages informels, comme savoir utiliser le lieu tout seul ou avec un adulte, ou savoir s'y comporter ; peut-être même, mais cela reste à évaluer, acquérir certaines notions de sciences.

Cependant, les résultats précédents sont évidemment à nuancer. En effet, pour que les objectifs des concepteurs soient atteints, parents et enfants doivent jouer le jeu de l'institution culturelle; les conditions de l'apprentissage sont elles aussi importantes. La prise en compte des interactions ${ }^{1}$ entre personnes montre que la coéducation fonctionne très inégalement. Ainsi, on distingue les quatre cas de figure suivants :

- la relation parent/enfant autre que le sien : certains parents jouent un rôle de régulateur entre les enfants. Certains profitent de leur statut d'adulte pour intimider les jeunes visiteurs qui ne sont pas avec eux et pour privilégier les enfants qu'ils accompagnent. C'est souvent le cas pour les activités individuelles comme la machine à boule. Dans ce cas, on ne peut pas véritablement parler d'apprentissage. L'inverse peut aussi se produire ; il n'est pas rare de voir des parents retrousser les manches d'enfants qu'ils ne connaissent pas

1 "L'interaction sociale au musée", Publics et Musées, Lyon, n 5, janvier-juin 1994. 
dans l'espace mains dans l'eau. Dans ce cas, l'apprentissage ne semble pas être perturbé ;

- la relation familiale parent/enfant : dans certains cas les parents se montrent trop directifs et, afin d'assouvir leur propre curiosité, ils vont même jusqu'à pousser l'enfant à faire et poursuivre une activité alors que celui-ci n'est pas encore prêt. Dans ce cas, l'objectif de plaisir, que les concepteurs ont sous-entendu en créant l'espace n'est pas respecté ; l'enfant entre de nouveau dans le circuit de l'apprentissage à tout prix. Mais ce cas n'est pas majoritaire ; bien souvent, les parents aident les enfants pour déchiffrer, les stimulent... et lorsqu'ils voient que les enfants sont autonomes, ils profitent pour faire à leur tour la découverte du lieu ;

- la relation enfant/enfant : ce cas peut être le plus enrichissant. Les relations sont, là encore, de 2 types :

- d'une part, des techniques d'intimidation entre enfants : "Tu peux te lever, c'était ma place". Et là, il n'est pas rare de voir un enfant, ayant cédé une ou deux fois à ses camarades, prendre de l'assurance et refuser catégoriquement ;

- d'autre part, l'entraide qui peut être du point de vue physique : un plus grand qui soulève un enfant qu'il ne connaît pas parce que ce dernier est trop petit pour voir ; c'est souvent le cas devant les aquariums ou la machine à boule. Cela concerne également une assistance verbale où un enfant explique comment il faut faire : "mais non c'est pas comme ça, regarde!". Dans ce second cas, on peut souvent voir ensuite ces enfants collaborer pendant un moment plus long. Ce qui à cet âge là n'est pas très commun, lorsque les enfants ne se connaissent pas ${ }^{1}$;

- la relation parent/parent : cette situation de coopération/concurrence semble importante puisqu'elle paraît jouer un rôle sur le comportement des enfants qui, probablement influencés par les adultes, reproduisent le même schéma qui est de deux sortes, soit l'intimidation, en période de forte affluence, soit l'entraide.

Ces quelques exemples révèlent que les conditions propres aux apprentissages non formels dans des établissements culturels existent (les enfants apprennent en expérimentant; ils ont normalement une liberté presque totale dans leur rythme de visite ; ils visitent de

1 A. Beaumatin, C. Laterasse, La psychologie de l'enfant, Toulouse, Éd. Milan, coll. "Les Essentiels de Milan", $n^{\circ} 86,1997$, pp. 36 et 57. 
manière autonome : les conditions sont réunies pour provoquer le plaisir des enfants qui peut faciliter les apprentissages non formels). Les résultats obtenus sont évidemment ponctuels. Ils doivent être confirmés ultérieurement par une recherche plus complète. Cependant, cette expérience est intéressante dans le sens où elle donne la parole aux enfants et autorise tout un jeu d'observations en rapport avec les apprentissages non formels. La seconde recherche qui a été conduite au Préau des Accoules à Marseille, en vue d'évaluer l'impact de tels apprentissages sur des tout-petits, a confirmé ces observations.

\section{Le Préau des Accoules, espace pour les enfants}

\subsection{Historique et fonctionnement}

Le Préau des Accoules est une institution culturelle, créée spécialement pour les enfants. Elle a ouvert ses portes en 1991 à l'initiative de la ville de Marseille. Cet espace, situé au cœur de la vieille ville dans le quartier du Panier, occupe un bâtiment du XVII ${ }^{\mathrm{e}}$ siècle.

Le Préau des Accoules a pour vocation de sensibiliser le jeune public et de lui faire découvrir le patrimoine, à travers trois expositions annuelles, sur divers thèmes proches des enfants (par exemple les Indiens, l'Afrique, la mer,...). Chacune de ces expositions permet de confronter les jeunes visiteurs à des objets authentiques qui sont empruntés, le plus souvent, aux différents musées de la région! ${ }^{1}$.

Cet espace fonctionne uniquement avec des animateurs qui proposent des activités renouvelées à chaque exposition. Dès leur arrivée, les enfants sont pris en charge par un médiateur qui va les guider un moment dans leur découverte. La séance d'animation se déroule en plusieurs phases :

- l'accueil où les animateurs présentent et expliquent le thème de l'exposition ;

- la visite, où les médiateurs confrontent les enfants aux objets exposés ;

1 C. Duncan, "Un point de vue : le musée considéré comme système de communication et les implications de ce système dans les programmes éducatifs muséaux", in A. DEVALLÉE, Vagues, une anthologie de la nouvelle muséologie, Tome 1, Paris-Mâcon, Éd. W, MNES, coll. "Muséologia", 1992, pp. 259 à 270. 
- les activités proposées par les animateurs pour poursuivre la visite sous forme de jeux, d'ateliers, de mises en situation par l'intermédiaire de différentes actions de médiation, pour que les enfants mettent à profit les connaissances fraîchement acquises ${ }^{1}$. Dans tous les cas, chaque animation est basée sur une participation active de l'enfant qui écoute, qui répond aux questions, qui touche certains objets, qui joue, qui agit.

Le public est composé de visiteurs scolaires (plutôt école maternelle 3 à 6 ans et école primaire 7 à 10 ans). Chaque visite dure généralement entre $1 \mathrm{~h}$ et $1 \mathrm{~h} 30$. Au-delà, les enfants sont fatigués et leur attention moins soutenue. Les apprentissages seraient alors peu profitables ${ }^{2}$. Les animateurs adaptent leur comportement en fonction de chaque groupe. Toutes les animations fonctionnent, toutefois, sur un principe unique : les enfants parviennent à mieux comprendre les choses lorsqu'ils partent de ce qu'ils connaissent et qu'ils expérimentent ou qu'ils voient d'autres enfants expérimenter devant eux. Cette manière de fonctionner, qui vise avant tout à un apprentissage de l'enfant par l'intermédiaire d'une activité plaisante et non conventionnelle, prend souvent le pas sur le contexte formel de la visite scolaire.

\subsection{Objectifs de l'institution}

Les objectifs, que les concepteurs/animateurs de ce lieu se sont fixés pour les enfants, sont simples :

- une familiarisation avec la notion de patrimoine,

- une expérience aussi plaisante et amusante que possible afin de motiver d'autres visites de musées,

- une visite avec une participation active des jeunes visiteurs ${ }^{3}$, en vue de mieux mémoriser les connaissances et apprentissages,

- enfin, le dernier objectif, plus spécifique à l'exposition Camoin, est, d'une part, familiariser l'enfant avec la peinture et, d'autre part, développer son esprit d'observation.

1 E. Calllet, O. Coppey, op. cit.

2 B. Poucet, M. VAN-PraET, op. cit.

${ }^{3}$ M. AllaRD, op. cit. 


\subsection{Enquête et résultats ${ }^{1}$}

Dans le cadre de la recherche menée au Préau des Accoules, l'exposition "Camoin au Balcon : un peintre et son monde" a servi de support ; elle présentait quatre tableaux du peintre marseillais Charles Camoin qui appartenait à la mouvance "fauve "(Lola à l'ombrelle jaune, 1920 - Fenêtre de l'atelier à Saint-Tropez, 1960 - L'étoile Vesper à la fenêtre de la salle à manger, 1962 - Saint-Tropez, le port au voilier vert, 1964). Cette exposition était ouverte aux enfants à partir de 4 ans. Et, le jeune public, pouvait, grâce à ce peintre, découvrir la peinture, les couleurs et la lumière, la mer... C'est en ce sens qu'une animation a été spécialement créée pour lui.

La recherche entreprise au Préau des Accoules voulait vérifier si le "musée" 2 a réellement un impact pédagogique sur les enfants de 4 à 6 ans et quelle est la nature de cet effet. A partir des objectifs des concepteurs de cette exposition et une fois l'âge des enfants défini, il a été possible d'élaborer un questionnaire adapté (testé préalablement auprès du jeune public). Ce questionnaire, construit sur des photographies, comporte des questions courtes et fermées, avec un vocabulaire simple en rapport avec des enfants de 4 à 6 ans, tout en conservant à l'esprit les apprentissages que les animateurs veulent transmettre :

- la socialisation de l'enfant grâce au musée, par la reconnaissance du lieu et des comportements à adopter ;

- l'acculturation de l'enfant grâce au musée, par la reconnaissance des tableaux et l'identification de certains détails.

Ce questionnaire été administré 149 fois, et ce un jour, une semaine, un mois après la visite des enfants au Préau, afin de mesurer l'impact pédagogique de cette exposition-animation dans le temps. Chaque jeune visiteur a répondu de manière individuelle. Les enfants de quatre écoles maternelles et d'un centre aéré, ont accepté de se prêter à cette évaluation. La plupart de ces enfants venaient dans un "musée" pour la première fois.

1 S. ROMANO, "Entre musées et enfants de 4 à 6 ans, une action utile ou non ?", $L a$ lettre de l'OCIM, $\mathrm{n}^{\circ} 60$, novembre-décembre 1998, pp. 3-9.

2 Le Préau des Accoules fonctionne comme un musée pour les expositions et la médiation. Mais il ne possède pas de collection et ne peut pas être considéré comme un musée à part entière mais plutôt comme un espace culturel destiné aux enfants. 
L'ensemble des résultats montre que les enfants ont réalisé un véritable apprentissage. Non seulement, le musée semble avoir eu un certain effet sur leur comportement qu'ils ont adapté en fonction de la spécificité du lieu, mais il semble aussi que cette visite ait permis aux enfants d'apprendre à s'intéresser à une exposition de peinture (cf. les paragraphes suivants).

\subsubsection{Socialisation ${ }^{1}$}

Les enfants de 4 à 6 ans, en se rendant au musée, sortent de leur cadre de référence usuel : la famille et l'école. Cette sortie leur permet d'évoluer dans un lieu dont ils n'ont pas l'habitude ; ils vont devoir apprendre et respecter les règles propres à ce lieu².

L'assimilation des règles de comportements nécessite la reconnaissance du lieu où il faut les mettre en œuvre. A cet effet, une série de six photographies de lieux publics différents, dont le Préau des Accoules, a été présentée aux enfants interrogés. Il en résulte que ces jeunes visiteurs ont reconnu le Préau à $96 \%$ et $41 \%$ des enfants ont même affirmé qu'ils voyaient le Préau des Accoules en regardant une photographie d'un musée des Beaux-Arts où étaient également accrochés des tableaux. Cette méprise laisse penser que certains enfants ont pris conscience, dans une certaine mesure, de la vocation de lieux qui sont relativement proches; ce qui peut dénoter une forme d'apprentissage.

Quant aux règles de comportement, les réponses indiquent que la majorité des enfants discrimine sans ambiguïté les énoncés correspondant à des conduites formellement interdites dans le musée. Sur la totalité des enfants interrogés, plus de $80 \%$ ont répondu que l'on ne peut pas y manger, ni toucher les tableaux; et à plus de $90 \%$ que l'on ne peut pas dormir au Préau des Accoules mais surtout courir dans un tel lieu.

Réciproquement, ils citent aisément ce que l'on peut effectivement faire dans un musée. Ainsi, près de $95 \%$ des enfants affirment que l'on peut voir des tableaux, $85 \%$ que l'on peut apprendre des choses en relation avec la lumière et les couleurs et $67 \%$ que l'on peut travailler dans une telle institution culturelle.

A. Beaumatin, C. Laterasse, op. cit., p. 64.

2 P. BouRdieu, "Les trois états du capital culturel", Actes de la recherche en sciences sociales, Paris, nov. 1979, pp. 3 à 6 . 
Des pourcentages aussi élevés semblent montrer que l'enfant évolue et adapte son comportement face à ce nouvel espace, notamment en fonction de chaque nouvelle expérience. Ainsi, à la question : "Est-ce qu'on peut s'amuser au Préau des Accoules ?" un enfant a même introduit une nuance au verbe s'amuser en répondant : "Oui, on peut s'amuser, mais on ne peut pas faire la foire !". De même, certains enfants, ayant parfaitement compris et assimilé les règles qui régissent un tel lieu, vont jusqu'à transgresser l'interdit dès lors qu'ils se ne sentent plus surveillés. Dès lors, les enfants courent fréquemment dans le Préau des Accoules ou encore essaient de s'imposer dans les jeux de leurs camarades alors qu'ils savent parfaitement que cela leur est interdit.

\subsubsection{Délectation}

Pour l'exposition Camoin, l'équipe de médiation a tenté d'apprendre aux enfants à se servir d'une exposition de peinture, en suscitant un contact visuel soutenu avec des ceuvres. L'enfant apprend-il à repérer les choses importantes en regardant les toiles et à organiser mentalement les informations reçues ${ }^{1}$ ?

Pour vérifier cela, une série de reproductions de six toiles leur a été présentée, dont les quatre peintures de Camoin exposées au Préau des Accoules et deux distracteurs : un tableau de Camoin non exposé et un tableau de Constable (artiste anglais du XIX ${ }^{\mathrm{e}}$ siècle) utilisant le thème identique de la mer. Puis, une épreuve de reconnaissance des œuvres leur a été proposée, suivie, lorsque le tableau est reconnu, d'une série de questions sur des détails présents dans la toile.

Les résultats montrent que les quatre toiles de Camoin ont été largement identifiées, avec environ $99 \%$ pour Lola à l'ombrelle jaune, $95 \%$ pour la Fenêtre de l'atelier à Saint-Tropez, $94 \%$ pour L'étoile Vesper à la fenêtre de la salle à manger et $89 \%$ pour Le port au voilier vert. En revanche, pour les deux distracteurs, les pourcentages indiquent que seule la toile de Constable a été bien distinguée à $87 \%$; celle de Camoin n'a pas été clairement distinguée car $76 \%$ des enfants ont déclaré l'avoir vue au Préau des Accoules. Les enfants semblent donc avoir assimilé le style de Camoin et d'après certaines

1 O. COPPEY, "Le musée non-lieu de formation", Média Scope, $\mathrm{n}^{\circ} 3$, Versailles, coll. "CRDP", nov. 1992, pp. 75-78. A. BADDELEY, "Les mémoires humaines", La recherche, $\mathrm{n}^{\circ} 267$, vol. 25, Paris, juillet-août 1994, pp. $730-735$. 
réponses, la démarche de l'enfant semble ici identique à celle adoptée pour la reconnaissance du lieu : ils mémorisent et reconnaissent des détails. Un enfant a dit même, devant Fenêtre ouverte à Cannes : "J'ai vu ce tableau au Préau, mais le fauteuil n'était pas à la même place".

L'analyse des données portant sur ces deux types de questions révèle l'importance du rôle de l'animateur. En effet, il y a une correspondance entre les réponses des enfants et les préférences de chaque médiateur vis-à-vis des toiles de Camoin. Ces résultats peuvent laisser apparaître que l'apprentissage est influencé par la passion exprimée par chaque animateur au cours de la visite-animation. Le fait que le médiateur influence l'apprentissage tend donc à nuancer l'idée que le musée, en lui-même, est un dispositif d'éducation non formelle. En effet que se passerait-il sans la présence de ce médiateur/éducateur ?

Incontestablement, les institutions culturelles dans la continuité de l'école contribuent à la socialisation de l'enfant. Tout en éprouvant du plaisir à travers les activités proposées, les enfants semblent avoir appris à utiliser "un musée". Ils se sont familiarisés avec la lumière, la peinture en général et, en particulier, avec celle de Charles Camoin. Ainsi, lors du dernier passage dans une école (un mois après la visite), de nombreux dessins étaient accrochés aux murs. Ils s'agissait de photocopies sur le thème du bord de mer, initialement en noir et blanc et coloriées par les enfants. Tous ces dessins présentaient des couleurs différentes et les enfants avaient même pris soin de traiter tout le dessin en tons clairs ou en tons foncés, sans toutefois les mélanger. La maîtresse a dit: "Lorsque je leur ai posé la question, pourquoi n'avaient-ils pas tous mis les mêmes couleurs ? Les enfants m'ont expliqué qu'ils avaient peint aux différents moments de la journée" de manière similaire aux effets rendus par le peintre Camoin. Les enfants de cette école ont su adapter les connaissances acquises avec l'exposition Camoin et prendre une distance par rapport à cette manifestation culturelle, en sachant réutiliser ce qu'elle leur avait apporté .

1 A. B ADDELEY, op. cit. 


\section{Conclusion}

Plusieurs pistes de recherche se dessinent à l'issue de cette double investigation. En premier lieu, il faut souligner la complexité et les exigences méthodologiques fortes de ce type de recherche. Une chose est de croire que l'action culturelle est bénéfique, tout autre chose est de se donner les moyens de la quantifier.

En second lieu, quand on parle d'apprentissage non formel, on pointe surtout l'idée d'une opposition entre deux milieux distincts : l'école et hors l'école. Mais il est évident que les apprentissages n'ont rien de spontané et qu'en préalable il y a des intentions éducatives et une préparation minutieuse d'intervenants, qui certes, ne sont pas des enseignants mais qui, comme eux, veulent agir.

En troisième lieu, il est patent que ce qui se passe dans les espaces non formels est infiniment plus complexe et ambigu que ce que laissent croire les affirmations à caractère idéologique sur le bien fondé de l'action culturelle. Qu'il s'agisse de l'explicitation des intentions éducatives des promoteurs de l'éducation non formelle, des dispositifs qu'ils construisent ou des actions qu'ils mettent en place, leurs représentations sont tout aussi généreuses et conformistes que ne le sont celles des enseignants. Et ils se donnent assez peu les moyens de vérifier et de contrôler leurs effets.

Les observations et la recherche menées dans des lieux précis, auprès des enfants comme des adultes qui les accompagnent, font apparaître de multiples interactions qui pèsent plus ou moins sur la nature des changements susceptibles d'être provoqués chez les enfants par leur participation à ces actions d'éducation non formelle. 